\title{
Association of CYP2B6 Genetic Variation with Efavirenz and Nevirapine Drug Resistance in HIV-I Patients from Botswana
}

This article was published in the following Dove Press journal: Pharmacogenomics and Personalized Medicine

\author{
Monkgomotsi J Maseng ${ }^{1,2}$ \\ Leabaneng Tawe ${ }^{1-3}$ \\ Prisca K Thami $\mathbb{D D}^{2,4}$ \\ Kaelo K Seatla $\mathbb{D}^{1,2}$ \\ Sikhulile Moyo $\mathbb{D}^{2,5}$ \\ Axel Martinelli ${ }^{6}$ \\ Ishmael Kasvosve' \\ Vladimir Novitsky ${ }^{2,5}$ \\ Max Essex ${ }^{2,5}$ \\ Gianluca Russo $\mathbb{D}^{7}$ \\ Simani Gaseitsiwe ${ }^{2,5}$ \\ Giacomo M Paganotti $\mathbb{1}^{3,8,9}$
}

'School of Allied Health Professions, Faculty of Health Sciences, University of Botswana, Gaborone, Botswana;

${ }^{2}$ Botswana-Harvard AIDS Institute Partnership, Gaborone, Botswana; ${ }^{3}$ Botswana-University of Pennsylvania Partnership, Gaborone, Botswana; ${ }^{4}$ Division of Human Genetics, Department of Pathology, Faculty of Health Sciences, University of Cape Town, Cape Town, South Africa; ${ }^{5}$ Department of Immunology and Infectious Diseases, Harvard School of Public Health, Boston, MA, USA; ${ }^{6}$ BigOmics Analytics, Bellinzona, Switzerland; ${ }^{7}$ Department of Public Health and Infectious Disease, Faculty of Medicine, Sapienza University of Rome, Rome, Italy; ${ }^{8}$ Division of Infectious Diseases, Perelman School of Medicine, University of Pennsylvania, Philadelphia, PA, USA; ' ${ }^{\circ}$ Department of Biomedical Sciences, Faculty of Medicine, University of Botswana, Gaborone, Botswana

Correspondence: Giacomo M Paganotti Botswana-University of Pennsylvania Partnership, PO Box AC I47 ACH,

Gaborone, Botswana

Tel +267 76416198

Email paganottig@bup.org.bw
Purpose: CYP2B6 liver enzyme metabolizes the two non-nucleoside reverse transcriptase inhibitors Efavirenz (EFV) and Nevirapine (NVP) used in the antiretroviral therapy (ART) regimens for $\mathrm{HIV}$-infected individuals. Polymorphisms of the CYP2B6 gene influence drug levels in plasma and possibly virological outcomes. The aim of this study was to explore the potential impact of CYP2B6 genotype and haplotype variation on the risk of developing EFV/NVP drug resistance mutations (DRMs) in HIV-1 patients receiving EFV-/NVPcontaining regimens in Botswana.

Patients and Methods: Participants were a sub-sample of a larger study (Tshepo study) conducted in Gaborone, Botswana, among HIV-infected individuals taking EFV/NVP containing ART. Study samples were retrieved and assigned to cases (with DRMs) and controls (without DRMs). Four single-nucleotide polymorphisms (SNPs) in the CYP2B6 gene $(-82 \mathrm{~T}>\mathrm{C} ; 516 \mathrm{G}>\mathrm{T} ; 785 \mathrm{~A}>\mathrm{G} ; 983 \mathrm{~T}>\mathrm{C})$ were genotyped, the haplotypes reconstructed, and the metabolic score assigned. The possible association between drug resistance and several independent factors (baseline characteristics and $C Y P 2 B 6$ genotypes) was assessed by Binary Logistic Regression (BLR) analysis. EFV/NVP resistance status and CYP2B6 haplotypes were also analyzed using Z-test, chi-square and Fisher's exact test statistics.

Results: Two hundred and twenty-seven samples were analysed (40 with DRMs, 187 without DRMs). BLR analysis showed an association between EFV/NVP resistance and CYP2B6 516G allele (OR: 2.26; 95\% CI: 1.27-4.01; $P=0.005$ ). Moreover, haplotype analysis revealed that the proportion of EFV/NVP-resistant infections was higher among CYP2B6 fast than extensive/slow metabolizers $(30.8 \%$ vs $16.8 \% ; P=0.035)$, with the $516 \mathrm{G}$ allele more represented in the haplotypes of fast than extensive/slow metabolizers $(100.0 \%$ vs $53.8 \%$; $P<0.001)$.

Conclusion: We demonstrated that the CYP2B6 516G allele, and even more when combined in fast metabolic haplotypes, is associated with the presence of EFV/NVP resistance, strengthening the need to assess the CYP2B6 genetic profiles in HIV-infected patients in order to improve the virologic outcomes of NNRTI containing ART.

Keywords: ART, CYP2B6 gene, drug resistance selection, fast metabolizers, HIV

\section{Introduction}

Antiretroviral therapy (ART) has significantly reduced HIV-related morbidity and mortality globally. However, the emerging threat of HIV drug resistance may reduce ART efficacy resulting in pronounced negative public health impact, especially in sub-Saharan Africa which account for about $70 \%$ of the global HIV epidemic. ${ }^{1}$ Scale-up of ART availability has been implemented and, in 2019, 
a global median of $67 \%$ of those in need $(90 \%$ in Botswana) $)^{2,3}$ have had access to ART. ${ }^{4}$ Among the antiretroviral drugs (ARVs) used as first-line ART regimen, first-generation Non-Nucleoside Reverse Transcriptase Inhibitors (NNRTIs), such as Efavirenz (EFV) and Nevirapine (NVP), are still largely used for HIV treatment in sub-Saharan Africa, ${ }^{5}$ although since 2017 the World Health Organization (WHO) has been recommending an EFV/NVP-sparing ART regimen in countries in which resistance to NNRTIs exceeds $10 \%{ }^{6}$ Despite the introduction of Dolutegravir (DTG)-based first-line ART in Botswana in June 2016, a significant proportion of HIV patients are still on EFV- or NVP-containing ART regimen. ${ }^{7}$ A recent (2019) WHO HIV drug resistance report encompassing 18 countries (6 from sub-Saharan Africa) revealed that pre-treatment HIV drug resistance rate to EFV/NVP exceeds $10 \%$ amongst adults initiating first-line ART (nearly twice as high in women than men). ${ }^{5}$ The rate was even higher (up to $30 \%$ ) in those previously exposed to ARVs, including women having taken ARVs for the prevention of mother-to-child transmission. ${ }^{5}$

Antiretroviral therapy efficacy largely depends on adequate drug exposure to suppress viral replication and allow the immune system to recover. However, occurrence of drug toxicity, suboptimal patient's compliance, suboptimal virologic responses, incomplete immune reconstitution and/or emergence of drug resistance limit therapeutic outcomes. ${ }^{8}$ HIV drug resistance, beside known viral factors, more frequently occurs because of sub-therapeutic ARV drug exposure and/or acquisition of drug-resistant strains. In resource-limited settings such as Botswana, HIV-diagnosed individuals with virologic failure are more likely to stay on virologically failing regimens for prolonged periods, because of lack of adequate virological follow-up. This may result in an ineffective drug exposure potentially causing drug toxicity and a higher risk of selecting and transmitting drug-resistant viruses. ${ }^{9}$ Moreover, the presence of HIV drug resistance mutations (DRMs) in minor viral populations is associated with an increased risk of virologic failure, in particular for NNRTI-based ART regimens, regardless of adherence, ethnicity, and immuno-virological basal characteristics of patients. ${ }^{8}$

Efavirenz and NVP are primarily metabolized in the liver by the cytochrome P450 2B6 enzyme (CYP2B6) with a minor contribution from other cytochromes (i.e. CYP2A6, CYP3A4/5). ${ }^{10-12}$ Studies on several populations have shown that Africans display the greatest level of genetic diversity in the CYP2B6 gene. ${ }^{13}$ Cytochrome P450 2B6 is one of the most polymorphic CYP450 genes in humans with over 100 described single nucleotide polymorphisms (SNPs), numerous complex haplotypes and distinct ethnic frequencies. ${ }^{14}$ Cytochrome P450 2B6 gene polymorphism has been associated with interindividual differences in drug pharmacokinetics and consequent plasma exposure, with possible consequences on drug efficacy and safety. ${ }^{14}$ There are different SNPs in the CYP2B6 gene that, according to their combination as haplotypes, may lead to different degrees of slow and/or fast EFV/NVP metabolizer phenotypes. ${ }^{14}$ Among those SNPs, 516G $>\mathrm{T}$ (rs3745274) and 983T $>\mathrm{C}$ (rs28399499) are associated with a significant loss of CYP2B6 function, leading to reduced clearance and prolonged half-life both for $\mathrm{EFV}^{15-18}$ and NVP. ${ }^{19,20}$ The $983 \mathrm{~T}>\mathrm{C}$ SNP affects the metabolism of both EFV and NVP; the 516G>T SNP influences mainly the EFV metabolism, while data on its impact on NVP metabolism are less conclusive. ${ }^{21,22}$ Conversely, two other SNPs, namely $785 \mathrm{~A}>\mathrm{G}$ (rs2279343) and $-82 \mathrm{~T}>\mathrm{C}$ (rs34223104) are associated with a gain of CYP2B6 function, leading to lower drug exposure. ${ }^{23-25}$ Indeed, the $785 \mathrm{~A}>\mathrm{G}$ SNP increases $\mathrm{EFV}^{26}$ and $\mathrm{NVP}^{27}$ metabolism, whereas there are no studies assessing the clinical/pharmacological impact in vivo of the $-82 \mathrm{~T}>\mathrm{C}$ SNP. Nonetheless, CYP2B6 $-82 \mathrm{~T}>\mathrm{C}$ has recently been included in the panel of CYP2B6 SNPs that should be considered for the evaluation of therapeutic impact by the Clinical Pharmacogenetics Implementation Consortium. $^{28}$

Pharmacogenetic studies of EFV and NVP have mostly been based on the CYP2B6 516G $>\mathrm{T}$ and 983T $>\mathrm{C}$ SNPs, with little or no clear assessment of the impact of the CYP2B6 $785 \mathrm{~A}>\mathrm{G}$ and $-82 \mathrm{~T}>\mathrm{C}$ SNPs. ${ }^{14}$ Studies from Botswana on HIV patients taking EFV-based ART showed that the CYP2B6 516T allele was protective against 1-year, ${ }^{29}$ but not at 6 -months, ${ }^{30}$ virologic breakthrough. However, no HIV DRMs have been assessed in either study. Similar results on the influence of the CYP2B6 $516 \mathrm{G}>\mathrm{T}$ substitution on virologic outcome were observed in studies from the US involving HIV patients of African ancestry $^{31}$ and HIV-diagnosed women from a multi-ethnic cohort, ${ }^{32}$ whereas other studies did not find any evidence of protection. ${ }^{33-35}$ Another work, on 66 HIV-diagnosed women from Kenya taking NVP-based ART, showed no associations of CYP2B6 516G $>\mathrm{T}$ and $983 \mathrm{~T}>\mathrm{C}$ with virologic response and toxicity at 12 months of follow-up. ${ }^{20}$ 
Study results concerning the association of CYP2B6 slow metabolizer profiles (defined by the presence of 516T and/or 983C alleles) and EFV and NVP-related adverse events and/ or toxicity are also conflicting, with some showing an association $^{15,36-39}$ and others not. ${ }^{18-20,30,31,40,41}$ Notably, due to the complexity of the CYP2B6 polymorphisms and the highest frequency of slow/intermediate genotypes among individuals of African ancestry, it is likely that haplotypes rather than a single polymorphism are better predictors of EFV/NVP plasma concentrations, ${ }^{14}$ as well as of toxicity, in which polymorphisms in genes other than the CYP450 system may also play a role. ${ }^{42,43}$

Efavirenz and NVP have a long half-life (estimated at 40-115 and 25-164hrs, respectively), a low genetic barrier for HIV drug resistance, and complex pharmacogenetics, which raises the possibility of sub-therapeutic drug concentration in plasma, especially among CYP2B6 fast metabolizers, an aspect that has not been fully studied. The CYP2B6 fast metabolizer profile may allow low EFV/NVP plasma exposure, possibly leading to the selection and spread of HIV mutations and consequent viral drug resistance. On the other hand, EFV/NVP CYP2B6 slow metabolizers are exposed to higher drug plasma concentration, leading to potential higher toxicity and consequently reduced patient's adherence and/or loss to care with possibility of sub-therapeutic plasma exposure and higher risk of HIV drug resistance (at least in a longterm perspective). While studies produced conflicting results, a posology adjustment according to the CYP2B6 polymorphism background has been proposed to address these potential issues in the context of personalized medicine. ${ }^{44,45}$

In summary, different $C Y P 2 B 6$ genotypes may influence immuno-virological response and/or toxicity by affecting EFV and NVP plasma concentration. ${ }^{14}$ We explored the possible impact of CYP2B6 genetic (and haplotype) variation on the risk of selection, accumulation and spread of HIV DRMs, providing a particular attention to the CYP2B6 fast metabolizer profile. To date, to the best of our knowledge, this aspect has not yet been fully evaluated.

This study was performed in Botswana with the aim to: i) assess $C Y P 2 B 6$ genotypes (for $\mathrm{SNPs}-82 \mathrm{~T}>\mathrm{C}, 516 \mathrm{G}>\mathrm{T}$, $785 \mathrm{~A}>\mathrm{G}, 983 \mathrm{~T}>\mathrm{C}$ ) in HIV-diagnosed adults taking EFV or NVP containing ART, and to classify corresponding CYP2B6 phenotypes as very slow, slow, extensive, rapid and ultra-rapid metabolizers; ii) construct haplotypes and apply a metabolic score according to the DRMs profile; iii) determine if there is any association of the CYP2B6 genotypes and haplotypes with the presence of EFV/NVPresistant infections.

\section{Patients and Methods Study Design and Sample Size}

This retrospective case-control study was part of a larger Tshepo study ${ }^{46}$ that was conducted at Botswana-Harvard AIDS Institute Partnership (BHP) between 2002 and 2007. The Tshepo study was a 5-years open-label, randomized study with a sample of 650 HIV-1 diagnosed ART naïve Botswana citizens (451 females and 199 males) attending the Infectious Disease Care Clinic (IDCC) in Princess Marina Hospital in Gaborone. The aim of Tshepo study was to evaluate the efficacy, tolerability and occurrence of drug resistance of six (6) different first-line ART regimens, all including an NNRTI either EFV or NVP, during a follow-up period of 156 weeks. For the purpose of the present study, being patient's adherence assessed and comparable results, ${ }^{46}$ cases were defined as HIV-diagnosed individuals taking EFV or NVP containing ART and having virological failure (evaluated after at least 4 months of ART) related to DRMs assessed by HIV genotyping, whereas controls were HIV-diagnosed individuals taking EFV or NVP-based ART without virological failure. ${ }^{46}$

Overall, 242 available residual samples were included in the present study. Of them, 40 were available cases that developed NNRTI resistance mutations, and 202 were controls.

\section{DNA Extraction}

Genomic DNA was extracted using Qiagen kits manual platform according to the manufacturer's protocol (Qiagen, Hilden, Germany) from about $200 \mu \mathrm{L}$ of peripheral blood mononuclear cells (PMBC's) stored at $-80^{\circ} \mathrm{C}$.

\section{CYP2B6 Genotyping}

CYP2B6 516G $>\mathrm{T}$ (rs3745274) detection was carried out using PCR-RFLP technique according to Lavandera et $\mathrm{al}^{47}$ protocol with minor modifications. CYP2B6 983T $>\mathrm{C}$ (rs28399499) detection was carried out using a touchdown PCR-RFLP assay published by Paganotti et al. ${ }^{48}$ CYP2B6 $785 \mathrm{~A}>\mathrm{G}$ (rs2279343) detection was done using an in-house optimized RFLP-PCR protocol. ${ }^{49}$

For purposes of this study, we also adopted a new inhouse assay for analysis of the CYP2B6 -82T $>\mathrm{C}$ (rs34223104) polymorphism. We designed two (2) primers (forward primer: 5'-CAAGCAGGAAGTCTGGGTTC-3'; reverse primer: 3'-AGTTCCATGGTCCTGGTCT-5'). PCR reaction was conducted in a total volume of $20 \mu \mathrm{L}$ containing 100ng genomic DNA. PCR protocol with the 
following conditions was used: 3 min of denaturation at $94{ }^{\circ} \mathrm{C}, 30$ s at $94{ }^{\circ} \mathrm{C}, 30$ s at $64{ }^{\circ} \mathrm{C}$ and 60 s at $72{ }^{\circ} \mathrm{C}$ for 35 cycles with a final step of $5 \mathrm{~min}$ at $72{ }^{\circ} \mathrm{C}$. The PCR product was then digested with $P$ siI restriction enzyme at $37{ }^{\circ} \mathrm{C}$ for $90 \mathrm{~min}$. The enzyme cuts the wild-type allele (T) in two fragments of $92 \mathrm{bp}$ and 88bp; while the mutant allele is not cut. The digested fragments were visualized on a $4 \%$ metaphor gel stained with ethidium bromide.

\section{Metabolic Score (MS) by Haplotypes and CYP2B6 Inferred Metabolic Phenotypes}

CYP2B6 genotype and haplotype information was translated into a measure of phenotype using the metabolic score (MS) system, ${ }^{49}$ already adopted as "activity score" for $C Y P 2 A 6,{ }^{50} C Y P 2 C 19,{ }^{51} C Y P 2 D 6,{ }^{52}$ and also consistent with Vujkovic et al, ${ }^{41}$ for $C Y P 2 B 6$. The MS translates composite genotype and/or haplotype information into a qualitative measure of phenotype. The scores are based on the algebraic sum of the individual allele values according to an additive model for CYP2B6. ${ }^{49}$ The MS was set conferring a -1 value for each slow metabolism alleles $(516 \mathrm{~T}, 983 \mathrm{C})$ and +1 for rapid metabolism alleles $(-82 \mathrm{C}$, $785 \mathrm{G})$, while an extensive metabolism allele was scored 0 $(-82 \mathrm{~T}, 516 \mathrm{G}, 785 \mathrm{~A}, 983 \mathrm{~T})$ both for composite CYP2B6 genotypes and haplotypes. $^{49}$

\section{Statistical Analysis}

Several methods were applied. We used the Arlequin software (v3.5.2.2) ${ }^{53}$ to test for Hardy-Weinberg Equilibrium (HWE) and the genetic fixation index $\left(\mathrm{F}_{\mathrm{ST}}\right)$ with default settings, while Linkage Disequilibrium (LD) was tested using the Expectation-Maximization (EM) algorithm with 20,000 permutations and three initial conditions. Binary
Logistic Regression (BLR) analysis (run on IBM SPSS statistical package, version 20) was applied to find any association between the dependent variable "drug resistance" with the independent variables (age, BMI, baseline CD4+ T-cell count and viral load, CYP2B6 genotype). Fisher's exact test, chi-square test and z-statistic were applied for statistical significance where needed.

\section{Results}

\section{Baseline Population Demographics}

Out of the 242 samples, 15 were excluded ( 5 due to the lack of complete genotypic information and 10 failed PCR). Thus, 227 samples were retained for analysis, with $40(18.6 \%)$ belonging to the group that developed virological failure with EFV/NVP DRMs. The remaining 187 samples $(81.4 \%)$ belonged to the group that did not develop virological failure during the follow-up period. Information about gender was available for 223 individuals, $146(65.5 \%)$ being females. The study population characteristics at baseline were available for 225 individuals, being as follows: mean age 33.7 years (range: 20.4-50.9); mean BMI 21.3 (range: 14.5-34.6); median baseline CD4+ T-cell count 188 cells/ $\mu \mathrm{L}$ (IQR: 147-221); median baseline viral load 5.30 $\log _{10}$ copies/ mL (IQR: 4.83-5.71). The ART regimen data were available for 225 individuals, with 117 (50.6\%) receiving EFV, and $108(46.8 \%)$ receiving NVP containing ART. Table 1 summarises the baseline characteristics of the study population according to the EFV/NVP resistance status.

\section{Virologic Failure and EFV/NVP Resistance}

This study was based on a subsample of the Tshepo study ${ }^{46}$ conducted in Botswana on HIV-diagnosed

Table I Baseline Characteristics of the Study Population

\begin{tabular}{|c|c|c|c|}
\hline Characteristics & Overall & EFV/NVP-Resistant & EFV/NVP-Susceptible \\
\hline Subjects, n (\%) & $227(100.0 \%)$ & $40(17.6 \%)$ & 187 (82.4\%) \\
\hline Females, n (\%) & I 46 (65.5\%)*, a & $27(67.5 \%) *$ & 119 (65.0\%)*, \\
\hline Males, n (\%) & 77 (34.5\%)*, & $13(32.5 \%)^{*}$ & $64(35.0 \%)^{*},{ }^{a}$ \\
\hline Mean age, years (range) & $33.7(20.4-50.9)$ & $34.7(20.4-50.9)$ & $33.5(22.9-49.6)$ \\
\hline Mean BMI (range) & $21.3(14.5-34.6)$ & $21.8(16.3-34.6)$ & $21.1(14.5-31.8)$ \\
\hline Median T-CD4, cells $/ \mu \mathrm{L}$ (IQR) & $188(|47-22|)$ & $194(97.5-241.5)$ & $187(|52.2-2| 9.0)$ \\
\hline Median Viral Load, $\log _{10}$ copies/mL (IQR) & $5.30(4.83-5.7 I)$ & $5.4 I(4.9 I-5.75)$ & $5.27(4.82-5.66)$ \\
\hline EFV-based ART, n (\%) & II $5(50.7 \%)^{\S}$ & $16(13.9 \%)^{\S}$ & $99(86.1 \%)^{\S}$ \\
\hline NVP-based ART, n (\%) & $107(47.1 \%)^{\S}$ & $24(22.4 \%)^{\S}$ & $83(77.6 \%)^{\S}$ \\
\hline Unspecified EFV/NVP-based ART, n (\%) & $5(2.2 \%)^{\S}$ & $0(0.0 \%)^{\S}$ & $5(100.0 \%)^{\S}$ \\
\hline
\end{tabular}

Notes: *Proportions calculated for columns; ${ }^{\S}$ proportions calculated for rows; ${ }^{a}$ gender data were not available for 4 individuals (all NNRTI-susceptible). Abbreviations: ART, antiretroviral therapy; NVP, nevirapine; EFV, efavirenz; BMI, body mass index; IQR, interquartile range. 
individuals taking EFV/NVP-based ART regimen and followed up to 3-years (156 weeks) after the treatment's start. All cases of virologic failure reported in the Tshepo study underwent HIV genotyping ${ }^{46}$ and EFV/NVP DRMs were detected in all the 40 cases used in the present study (Supplementary Table 1). Thus, in the present study, virologic failure and EFV/NVP DRMs coincide. ${ }^{46}$ The median interval between start of ART and appearance of virologic failure was 72 weeks (IQR: 45.5-104).

\section{CYP2B6 Genotype and Allele Frequencies}

The CYP2B6 genotype distribution and allele frequencies of the four SNPs $(-82 \mathrm{~T}>\mathrm{C}, 516 \mathrm{G}>\mathrm{T}, 785 \mathrm{~A}>\mathrm{G}, 983 \mathrm{~T}>\mathrm{C})$ are summarized in Table 2. The comparisons of the three genotypes distribution between the subjects with and those without HIV DRM for each single SNP were all not statistically different but for the CYP2B6 516G $>\mathrm{T}$ polymorphism $($ chi-square $=8.121 ; P=0.017)($ Figure 1$)$, with the wild-type extensive metaboliser $516 \mathrm{G}$ allele at higher frequency among resistant than sensitive infections (70\% vs $54 \%$, calculated from Table 2 ).

\section{Hardy-Weinberg Equilibrium and Linkage Disequilibrium Analysis}

Hardy-Weinberg Equilibrium analysis showed that CYP2B6 983 locus displayed significant deviations from HWE in EFV/NVP-resistant, EFV/NVP-susceptible and combined (overall) samples $(P=0.001 ; P<0.001 ; P<0.001$, respectively). A reason for this deviation may be due to a defect in heterozygous samples. Furthermore, CYP2B6 -82 and $C Y P 2 B 6516$ did not show a statistically significant deviation from HWE in the EFV/NVP-resistant HIV infections $(P=$ 0.449 and $P=0.230$, respectively), whereas a statistically significant deviation from HWE was noted in the EFV/NVPsusceptible $(P=0.039$ and $P=0.031$, respectively) and all the samples combined $(P=0.030$ and $P=0.010$, respectively), with an excess of heterozygotes in both groups. CYP2B6 785 genotypes were in equilibrium in all the groups analysed (EFV/NVP-resistant, EFV/NVP-susceptible and both combined).

Linkage disequilibrium was observed between CYP2B6 -82 and CYP2B6 516 in all groups (Table 3). Linkage disequilibrium was also observed between CYP2B6 -82 and CYP2B6 983, as well as between CYP2B6 516 and CYP2B6 983, in the EFV/NVPsusceptible and overall samples, but not in the EFV/NVPresistant group (Table 3). Finally, CYP2B6 785 did not

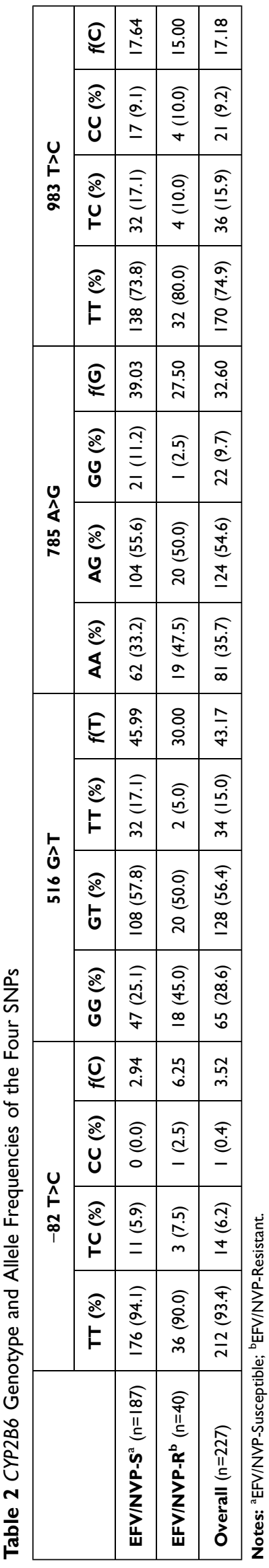




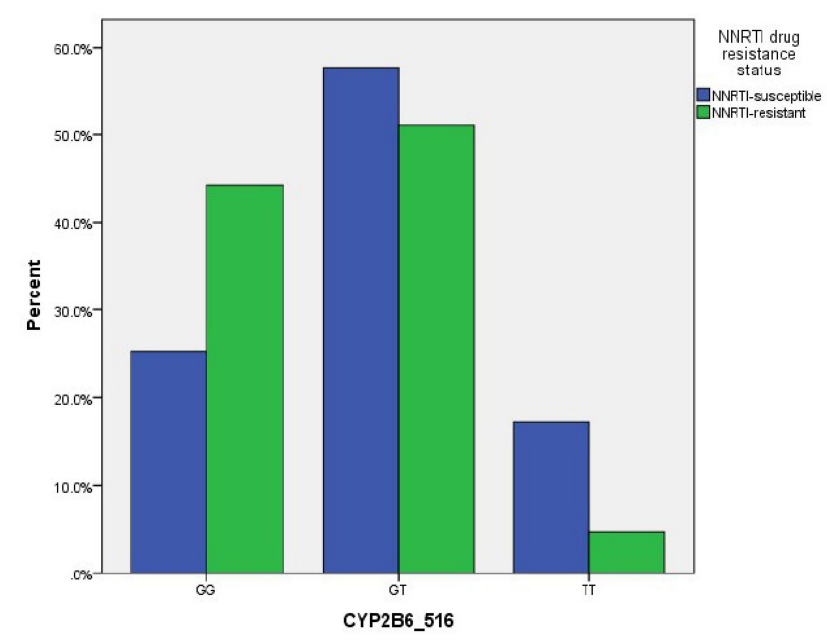

Figure I Distribution of CYP2B6-5/6 genotypes according to NNRTI-resistance status. Chi-square associated $P$-value is 0.017 .

show strong association with the other three loci, thus justifying the respect of $\mathrm{HWE}$ in all the three groups (EFV/NVP-resistant, EFV/NVP-susceptible, overall samples) (Table 3 ).

\section{Population Differentiation Tests}

The fixation index $\left(\mathrm{F}_{\mathrm{ST}}\right)$ between EFV/NVP-resistant and susceptible samples gave a distance of $0.023(P=0.008 \pm$

Table 3 Pairwise Linkage Disequilibrium (LD) Analysis for the Four Polymorphic Loci (CYP2B6 -82, 516, 785, 983)

\begin{tabular}{|l|c|c|}
\hline Phenotype & Comparison & P-value \\
\hline EFV/NVP-Resistant & 785 vs -82 & 0.054 \\
EFV/NVP-Resistant & 785 vs 516 & 0.882 \\
EFV/NVP-Resistant & 785 vs 983 & 0.195 \\
EFV/NVP-Resistant & $-\mathbf{8 2}$ vs $\mathbf{5 1 6}$ & $\mathbf{0 . 0 0 1}$ \\
EFV/NVP-Resistant & -82 vs 983 & 0.078 \\
EFV/NVP-Resistant & 516 vs 983 & 0.118 \\
EFV/NVP-Susceptible & $\mathbf{7 8 5}$ vs -82 & 0.513 \\
EFV/NVP-Susceptible & $\mathbf{7 8 5}$ vs 516 & 0.095 \\
EFV/NVP-Susceptible & $\mathbf{7 8 5}$ vs $\mathbf{9 8 3}$ & 0.685 \\
EFV/NVP-Susceptible & $-\mathbf{8 2}$ vs $\mathbf{5 1 6}$ & $<\mathbf{0 . 0 0 1}$ \\
EFV/NVP-Susceptible & $\mathbf{- 8 2}$ vs $\mathbf{9 8 3}$ & $\mathbf{0 . 0 0 1}$ \\
EFV/NVP-Susceptible & $\mathbf{5 1 6}$ vs $\mathbf{9 8 3}$ & $\mathbf{0 . 0 2 3}$ \\
Overall & $\mathbf{7 8 5}$ vs -82 & 0.221 \\
Overall & $\mathbf{7 8 5}$ vs 516 & 0.254 \\
Overall & $\mathbf{7 8 5}$ vs 983 & 0.324 \\
Overall & $-\mathbf{8 2}$ vs $\mathbf{5 1 6}$ & $<\mathbf{0 . 0 0 1}$ \\
Overall & $\mathbf{- 8 2}$ vs $\mathbf{9 8 3}$ & $<\mathbf{0 . 0 0 1}$ \\
Overall & $\mathbf{5 1 6}$ vs $\mathbf{9 8 3}$ & $\mathbf{0 . 0 0 6}$ \\
\hline
\end{tabular}

Notes: $P$-values for LD analysis were obtained using Arlequin. Significant comparisons are highlighted in bold.

Abbreviations: EFV, efavirenz; NVP, nevirapine.
0.003, 1023 permutations). While significant, this difference is consistent with panmixia between the two groups. Furthermore, an exact test of population differentiation revealed no statistical difference between the two groups ( $P=0.060 \pm 0.008 ; 100,000$ Markov chain steps).

\section{Binary Logistic Regression Analysis and EFV/NVP Resistance According to CYP2B6 Genotypes}

Binary Logistic Regression analysis was set with the dependent dichotomic variable assuming two values according to the presence of EFV/NVP-susceptible or EFV/NVP-resistant HIV infections. Factors tested were age, BMI, baseline lymphocytes T-CD4+, viral load, genotypes for $C Y P 2 B 6-82$, CYP2B6 516, CYP2B6 785, CYP2B6 983 (see Supplementary Table 2). First step results showed only one significant factor (CYP2B6 516G). After removal of the nonsignificant factors, the BLR was repeated with only one (significant) factor in the model (CYP2B6 516G $>\mathrm{T}$ ). This second step analysis revealed a significant statistical association between presence of CYP2B6 516G and DRMs status (OR: 2.26; 95\% CI:1.27-4.01; $P=0.005$ ).

Finally, the CYP2B6 516T allele presence (GT and TT genotypes) was tested for its possible protection against virologic failure (corresponding to EFV/NVP resistance in the present study as stated in the Methods section): no association was found at 6 months, nor beyond six months (up to 3 years) (Fisher's exact test, $P>0.05$ ).

\section{CYP2B6 Haplotype Frequency Estimation in EFV/NVP Susceptible and Resistant Groups} Haplotype frequencies for CYP2B6 were estimated using the EM algorithm in Arlequin (Table 4). For all the samples, based on the four SNPs of CYP2B6 analysed $(-82 \mathrm{~T}>\mathrm{C}, 516 \mathrm{G}>\mathrm{T}$, $785 \mathrm{~A}>\mathrm{G}, 983 \mathrm{~T}>\mathrm{C}$ ), the TGAT haplotype was the most common, whereas the CGAC, CTAT and CGGT haplotypes the rarest (Table 4). The haplotype TGAT was also the most abundant haplotype when EFV/NVP-resistant and susceptible samples were analysed separately, whereas no CGGT haplotypes were estimated in the EFV/NVP-susceptible group, and no TGGC, CGAC and CTAT haplotypes were estimated in the EFV/NVP-resistant group (Table 4).

\section{Metabolic Score by CYP2B6 Haplotype and EFV/NVP Resistance Status}

The inferred MS was calculated for the haplotypes and they are shown in Table 4. In order to assess the possible risk of 
Table 4 Estimated and Maximum-Likelihood (ML) Haplotype Frequencies by Phenotype and for All the Samples Combined. MaximumLikelihood Haplotype Frequencies are Shown in Parenthesis with Their Standard Deviations (SD). The Order of Nucleotides in the Reconstructed Haplotypes is Made According to the SNP Position in the CYP2B6 Gene (-82T>C, 5I6G>T, 785A>G, 983T>C)

\begin{tabular}{|c|c|c|c|c|c|}
\hline \multirow[t]{2}{*}{ Haplotype } & \multirow[t]{2}{*}{ MS } & \multirow[t]{2}{*}{ Phenotype } & \multicolumn{3}{|c|}{ Estimated and ML Frequencies \pm SD } \\
\hline & & & Overall* & EFV/NVP- Resistant & EFV/NVP-Susceptible \\
\hline TGAT & $\mathbf{0}$ & Extensive & $20 \mathrm{I}(0.44 \mathrm{I} \pm 0.023)$ & $43(0.526 \pm 0.065)$ & $162(0.430 \pm 0.029)$ \\
\hline TGAC & -1 & Slow & $24(0.054 \pm 0.013)$ & $5(0.065 \pm 0.035)$ & $19(0.052 \pm 0.015)$ \\
\hline CGAT & $\mathbf{I}$ & Rapid & $12(0.019 \pm 0.008)$ & $3(0.039 \pm 0.024)$ & $5(0.008 \pm 0.006)$ \\
\hline CGGT & 2 & Ultra-rapid & $2(0.004 \pm 0.003)$ & $2(0.024 \pm 0.018)$ & $0(0.000 \pm 0.000)$ \\
\hline TTAT & -1 & Slow & $37(0.078 \pm 0.015)$ & $6(0.082 \pm 0.036)$ & $27(0.073 \pm 0.017)$ \\
\hline TTAC & -2 & Very slow & $10(0.026 \pm 0.010)$ & I $(0.013 \pm 0.017)$ & $9(0.030 \pm 0.011)$ \\
\hline TGGT & $\mathbf{I}$ & Rapid & $16(0.041 \pm 0.011)$ & $3(0.046 \pm 0.023)$ & $13(0.041 \pm 0.011)$ \\
\hline TTGC & -1 & Slow & $4 \mathrm{I}(0.083 \pm 0.015)$ & $6(0.073 \pm 0.032)$ & $35(0.085 \pm 0.016)$ \\
\hline TTGT & 0 & Extensive & $107(0.236 \pm 0.020)$ & II $(0.132 \pm 0.046)$ & $96(0.252 \pm 0.026)$ \\
\hline TGGC & $\mathbf{0}$ & Extensive & $2(0.006 \pm 0.005)$ & $0(0.000 \pm 0.000)$ & $2(0.006 \pm 0.006)$ \\
\hline CGAC & $\mathbf{0}$ & Extensive & $\mathrm{I}(0.002 \pm 0.003)$ & $0(0.000 \pm 0.000)$ & I $(0.003 \pm 0.003)$ \\
\hline CTAT & 0 & Extensive & I $(0.009 \pm 0.009)$ & $0(0.000 \pm 0.000)$ & $5(0.013 \pm 0.006)$ \\
\hline CTGT & I & Rapid & $0(0.000 \pm 0.000)$ & $0(0.000 \pm 0.000)$ & $0(0.005 \pm 0.005)$ \\
\hline
\end{tabular}

Notes: *The combination of susceptible and resistant dataset does not necessarily result in a "sum" of the two haplotype counts estimated separately. Different sample counts in each group have an effect on the accuracy of the estimates.

Abbreviations: MS, metabolic score; N/A, not applicable; NC, not calculable (because of lack of estimated haplotype frequency).

carrying EFV/NVP-resistant HIV infections according to the CYP2B6 fast metabolizer profile, the metabolic phenotypes were classified as follows: $\mathrm{MS} \leq 0$, this including extensive, slow and very slow inferred metabolic phenotypes; $M S \geq 1$, this including rapid and ultra-rapid inferred metabolic phenotypes. The breakdown of metabolic phenotypes between groups according to the EFV/NVP resistance status are shown in Table 5. The comparison between EFV/NVPresistant and susceptible HIV infections by MS was associated to a $z$-statistic of $1.812(P=0.035)$, therefore showing that the rate of EFV/NV resistance was significantly higher among fast metabolizers haplotypes compared to the other group (30.8\% vs $16.8 \%$, respectively).

In line with the BLR results, the CYP2B6 516G allele was present in $100 \%(n=30 / 30)$ of rapid (CGAT and

Table 5 EFV/NVP Resistance by CYP2B6 Metabolic Phenotype

\begin{tabular}{|l|c|c|}
\hline \multirow{2}{*}{ EFV/NVP Resistance Status } & \multicolumn{2}{|c|}{$\begin{array}{c}\text { EFV/NVP Metabolic } \\
\text { Phenotype }\end{array}$} \\
\cline { 2 - 3 } & MS $\leq \mathbf{0}^{\mathbf{a}}$ & MS $\geq \mathbf{~ I}^{\mathbf{b}}$ \\
\hline Resistant, $\mathrm{n}(\%)$ & $72(16.8 \%)$ & $8(30.8 \%)$ \\
Susceptible, $\mathrm{n}(\%)$ & $356(83.2 \%)$ & $18(69.2 \%)$ \\
Total & $428(100 \%)$ & $26(100 \%)$ \\
\hline
\end{tabular}

Notes: Resistance and susceptible haplotypes were counted according to MS as from Table 4. The $z$ statistic is 1.812 . The one-tailed $P$-value is 0.035 . a MS $\leq 0$ : extensive, slow and very slow EFV/NVP metabolizers. ${ }^{\mathrm{b}} \mathrm{MS} \geq \mathrm{I}$ : rapid and ultra-rapid EFV/NVP metabolizers.
TGGT) and ultra-rapid (CGGT) haplotypes, whereas it was only present in $53.8 \%(n=228 / 424)$ of extensive/ slow metabolizers haplotypes (Fisher's exact test, $\mathrm{P}<$ 0.001) (see Table 4, “overall” column).

\section{Discussion}

Understanding the factors that modulate the selection of DRMs associated with HIV-1 infection is essential to design efficient control strategies. Drug resistance usually emerges rapidly when ARV drugs are administered as monotherapy or in the presence of incomplete viral suppression, suggesting that resistance is caused by the selection of mutant viruses within the host. ${ }^{54}$ Besides known viral factors (HIV diversity, HIV replication, drug selection pressure and fitness of drug-resistant viral subpopulations) and patient ART adherence, human genetic background is a possible further, not yet fully understood, co-factor affecting HIV drug resistance selection. In this study, we addressed the hypothesis that human pharmacogenetics can drive the selection of HIV drug resistance. We therefore found a statistically significant association between EFV/NVP-resistant HIV infections and CYP2B6 516G allele presence (OR: 2.26; 95\% CI:1.27-4.01; $P=$ 0.005). In fact, EFV/NVP resistant infections had higher $516 \mathrm{G}$ allele frequency (Table 2 and Figure 1). Further information comes from the haplotype reconstruction where $100 \%$ of rapid (CGAT and TGGT) and ultra-rapid 
Table 6 CYP2B6 SNPs $(-82 T>C, 516 G>T, 785 A>G, 983 T>C)$ Frequency in Sub-Saharan Africa

\begin{tabular}{|c|c|c|c|c|c|c|}
\hline \multirow[t]{2}{*}{ Geographic Region/Ethnic Populations } & \multirow[t]{2}{*}{ Number of Participants } & \multicolumn{4}{|c|}{ CYP2B6 SNPs Frequency (\%) } & \multirow[t]{2}{*}{ References } \\
\hline & & $-82 C$ & $516 \mathrm{~T}$ & $785 G$ & $983 \mathrm{C}$ & \\
\hline \multicolumn{7}{|l|}{ Southern Africa } \\
\hline Botswana & 101 & - & 36.6 & - & - & {$[57]$} \\
\hline Botswana & 1101 & - & 37.6 & - & - & [29] \\
\hline Botswana & 731 & - & - & 6.0 & 11.0 & [39] \\
\hline Botswana & 570 & - & 38.1 & 33.0 & 13.5 & [49] \\
\hline Malawi & 150 & - & 40.5 & 37.1 & 8.6 & [19] \\
\hline Mozambique & 105 & - & 34.7 & 44.2 & 8.6 & [39] \\
\hline Mozambique & 360 & - & 42.6 & 40.9 & - & [58] \\
\hline South Africa & 122 & - & 32.0 & - & - & [59] \\
\hline South Africa & 80 & - & 43.1 & - & - & [37] \\
\hline South Africa & 160 & - & 36.2 & 36.2 & 2.5 & {$[60,61]$} \\
\hline South Africa & 295 & - & 41.1 & 41.1 & 7.1 & {$[61]$} \\
\hline South Africa & 113 & - & 36.0 & - & 7.0 & [62] \\
\hline South Africa & 81 & - & 35.2 & 35.2 & 3.7 & [63] \\
\hline South Africa & 60 & - & 41.0 & 40.8 & 11.0 & [64] \\
\hline Zimbabwe & 36 & - & 51.4 & 52.8 & 11.1 & [65] \\
\hline Zimbabwe & 71 & - & 48.6 & - & - & [66] \\
\hline Zimbabwe & 49 & - & 41.8 & 41.8 & 9.1 & [67] \\
\hline Zimbabwe & 185 & - & 43.8 & - & 15.9 & [68] \\
\hline \multicolumn{7}{|l|}{ West-Central Africa } \\
\hline Cameroon & 69 & - & 36.9 & 32.6 & - & [60] \\
\hline Cameroon & 168 & - & 44.3 & - & 12.8 & [48] \\
\hline Cameroon & 122 & - & 59.4 & - & 8.6 & [69] \\
\hline Ghana & 40 & 1.2 & 48.8 & 47.5 & 6.6 & [70] \\
\hline Ghana & 42 & - & 54.0 & 46.0 & 7.6 & {$[71,72]$} \\
\hline Ghana & 705 & - & 48.0 & - & 4.0 & {$[18]$} \\
\hline Ghana & 94 & - & - & - & 4.2 & [42] \\
\hline Ghana & 74 & - & 44.6 & - & 4.6 & [73] \\
\hline Guinea & 21 & - & 50.0 & 48.0 & 1.6 & {$[71,72]$} \\
\hline Ivory Coast & 41 & - & 40.0 & 38.0 & 5.5 & {$[7 \mid, 72]$} \\
\hline Nigeria & 300 & - & 36.5 & - & - & {$[74]$} \\
\hline Nigeria & 77 & - & 43.7 & - & 13.2 & {$[75]$} \\
\hline Republic of Congo & 288 & - & 55.0 & - & - & {$[76]$} \\
\hline Sierra Leone & 52 & - & 47.0 & 36.0 & 3.8 & {$[71,72]$} \\
\hline \multicolumn{7}{|l|}{ East Africa } \\
\hline Burundi & 202 & - & 31.6 & - & 6.9 & [77] \\
\hline Ethiopia & 163 & - & 29.7 & - & - & [78] \\
\hline Ethiopia & 245 & - & 31.4 & - & - & [79] \\
\hline Ethiopia & 264 & - & 31.4 & - & - & [80] \\
\hline Ethiopia & 298 & - & 29.2 & - & - & {$[8 I]$} \\
\hline Kenya & 66 & - & 32.6 & - & 9.8 & [20] \\
\hline Rwanda & 80 & - & 31.9 & 32.5 & 9.2 & [17] \\
\hline Rwanda & 39 & 6.4 & - & - & - & [82] \\
\hline Rwanda & 90 & - & 32.8 & - & 8.0 & [83] \\
\hline Tanzania & 183 & - & 41.8 & - & - & {$[80]$} \\
\hline Tanzania & 242 & - & 36.0 & - & - & [84] \\
\hline Tanzania & 251 & - & 35.6 & - & 19.8 & [85] \\
\hline
\end{tabular}


Table 6 (Continued).

\begin{tabular}{|l|c|c|c|c|c|c|}
\hline Geographic Region/Ethnic Populations & Number of Participants & \multicolumn{3}{|c|}{ CYP2B6 SNPs Frequency (\%) } & \multicolumn{2}{c|}{ References } \\
\cline { 3 - 6 } & & & $-82 C$ & $\mathbf{5 1 6 T}$ & $\mathbf{7 8 5 G}$ & $\mathbf{9 8 3 C}$ \\
\hline Tanzania & 91 & - & 33.5 & - & 9.3 & {$[86]$} \\
Tanzania & 37 & - & 33.8 & - & - & {$[87]$} \\
Uganda & 23 & - & 30.4 & - & - \\
Uganda & 187 & - & 31.8 & - & - & {$[88]$} \\
Uganda & 74 & - & 29.1 & 32.4 & 5.4 & {$[65]$} \\
Uganda & 166 & - & 39.4 & - & - & {$[90]$} \\
Uganda & 57 & - & 33.3 & - & 8.8 & {$[91]$} \\
\hline
\end{tabular}

(CGGT) CYP2B6 haplotypes carried the 516G allele (Table 4), whereas only $53.8 \%$ of the extensive, slow (TGAC, TTAT and TTGC) and very slow (TTAC) haplotypes carried it. Moreover,

CYP2B6 rapid and ultra-rapid metabolizers showed a significantly higher frequency of EFV/NVP-resistant HIV infections than extensive and slow metabolizers $(30.8 \%$ vs $16.8 \% ; z$-statistic $=1.812 ; P=0.035)$, based on their haplotype reconstruction (Table 5). Nevertheless, it is important to note that the rate of rapid and ultra-rapid haplotypes is less than $6 \%(26 / 454)$ of the total haplotypes.

A possible interpretation of these results is that EFV/ NVP resistance tends to accumulate based on the presence of the CYP2B6 516G allele (GG>GT>TT), and that in rapid and ultra-rapid metabolisers might happen at a higher rate. This could be an important model for drug resistance selection that may be verified on a larger longitudinal cohort and tested on different pathogens, different antimicrobial drugs and epidemiological contexts.

These results are partially in line with similar findings on malaria drug resistance where it has been demonstrated that the $C Y P 2 C 8$ slow metabolizer phenotype is associated with the risk of carrying chloroquine- and amodiaquineresistant parasites. ${ }^{55,56}$

We also found that LD into the $\simeq 1000$ bp region analysed (spanning from CYP2B6 -82 to 983 nucleotide positions) was strong $(P<0.001)$ and departures from the HWE were coherent with the physical and genetic linkage between SNPs. Genotype and allele frequencies for the four CYP2B6 SNPs analysed in this study were in line with literature, including most if not all studies from Botswana (Table 6). The only discrepancy was represented by a study ${ }^{41}$ conducted in the same area as the present research that indicated an allele frequency for $C Y P 2 B 6$ $785 \mathrm{G}$ of $6 \%$, well outside the accepted range of
$35.2-52.8 \%$ for Southern Africa (Table 6). Finally, the two groups (with and without EFV/NVP-resistant HIV infections) into which we subdivided our study population appeared to be in panmixia, therefore offering an ideal situation for the comparison.

In summary, subjects carrying the CYP2B6 516G allele were more likely to carry HIV drug-resistant infections. Coherently with our hypothesis, the $516 \mathrm{G}$ allele is always present in the rapid and ultra-rapid haplotypes, confirming the possibility that rug resistance selection is enhanced when drug metabolism is faster. However, the rate of fast metabolisers was not high in this cohort, therefore reducing its possible impact.

The present study has few limitations: i) the sample size was relatively small, therefore haplotypes reconstruction provided higher statistical power in the comparisons, ii) it would have been more powerful to use matched-case control, to avoid any confounding factors, and iii) the lack of data on EFV/NVP plasma exposure hampered the quantitative confirmation of CYP2B6 metabolic phenotypes. Nonetheless, our findings suggest a trend towards a role for the genetic background of patients affecting drug therapy outcomes, and warrants further studies.

\section{Conclusion}

In conclusion, this work indicates that the CYP2B6 516G allele, and its combination into rapid and ultra-rapid metabolizer profiles, as defined by the correspondent haplotypes, is directly associated with the risk of development of drug resistance in HIV-diagnosed individuals receiving EFV- or NVP-containing ART in Botswana. However, larger studies will be needed to confirm this association. In general, our findings support the hypothesis that pharmacogenetics may play a significant role in HIV therapy outcomes. Besides the known possible 
impact of slow EFV/NVP metabolism on ART toxicity and compliance, fast EFV/NVP metabolism may also affect ART outcomes. A deeper knowledge of the genetic background at an individual level could thus be highly beneficial in personalising ART therapies and improving their efficacy, especially in patients who show poor response following initiation of treatment.

\section{Ethics Approval and Informed Consent}

This was a retrospective case-control study approved by Health Research Division Office (HRDC) of the Botswana Ministry of Health and Wellness. The approval was done in accordance with the amendments made to the initial permit of "The host genetics of HIV-1 subtype C infection progression and treatment in Africa/Gwas on determinants of HIV-1 subtype C infection" [Reference No: HPDME 13/18/1 X1 (163)]. For the purpose of this study signed informed consent was sought from the participants. In addition, Botswana-Harvard AIDS Institute Partnership, as the database owner authorized by HRDC, gave permission to use its data and samples for the current study. This study was conducted in accordance with the Declaration of Helsinki.

\section{Acknowledgments}

We would like to express our gratitude to SANTHE for funding this research in collaboration with Botswana Harvard AIDS Institute Partnership. We also express gratitude to the University of Botswana, Faculty of Health Sciences, School of Allied Health Professions and Botswana-University of Pennsylvania Partnership laboratory staff for their help, assistance and continuous support to this study. These authors have joint senior authorship: Gianluca Russo, Simani Gaseitsiwe, Giacomo M Paganotti.

\section{Funding}

This work was supported through the Sub-Saharan African Network for TB/HIV Research Excellence (SANTHE), a DELTAS Africa Initiative [grant \# DEL-15-006]. The DELTAS Africa Initiative is an independent funding scheme of the African Academy of Sciences (AAS)'s Alliance for Accelerating Excellence in Science in Africa (AESA) and supported by the New Partnership for Africa's Development Planning and Coordinating Agency (NEPAD Agency) with funding from the Wellcome Trust [grant \# 107752/Z/15/Z] and the UK government. The views expressed in this publication are those of the author(s) and not necessarily those of AAS, NEPAD Agency, Wellcome Trust or the UK government. The work was also supported by the Penn Center for AIDS Research [grant \# P30 AI045008].

\section{Disclosure}

The authors report no conflicts of interest in this work.

\section{References}

1. UNAIDS, 2020. UNAIDS data. 2020. Available from: https://www. unaids.org/sites/default/files/media_asset/2020_aids-data-book_en. pdf. Accessed March 8, 2021.

2. Makhema J, Wirth KE, Pretorius Holme M, et al. Universal testing, expanded treatment, and incidence of HIV infection in Botswana. N Engl J Med. 2019;381(3):230-242. doi:10.1056/NEJMoa1812281

3. Essex M, Makhema J, Lockman S. Reaching 90-90-90 in Botswana. Curr Opin HIV AIDS. 2019;14(6):442-448. doi:10.1097/COH.0000 000000000580

4. WHO, World Health Organization. Antiretroviral therapy coverage estimates by WHO region. 2020. Available from: https://apps.who. int/gho/data/view.main.23300REGION?lang=en. Accessed September $15,2020$.

5. WHO, World Health Organization. HIV drug resistance report 2019. 2019. Available from: https://www.who.int/hiv/pub/drugresistance/ hivdr-report-2019/en/. Accessed September 15, 2020.

6. WHO, World Health Organization. Guidelines for the public heath response to pretreatment HIV drug resistance. 2017. Available from: https://www.who.int/hiv/pub/guidelines/hivdr-guidelines-2017/en/. Accessed September 15, 2020.

7. Republic of Botswana. Handbook of the Botswana 2016 Integrated HIV Clinical Care Guidelines. 2016.

8. Li JZ, Paredes R, Ribaudo HJ, et al. Low-frequency HIV-1 drug resistance mutations and risk of NNRTI-based antiretroviral treatment failure: a systematic review and pooled analysis. JAMA. 2011;305(13):1327-1335. doi:10.1001/jama.2011.375

9. Cambiano V, Bertagnolio S, Jordan MR, Lundgren JD, Phillips A. Transmission of drug resistant HIV and its potential impact on mortality and treatment outcomes in resource-limited settings. $J$ Infect Dis. 2013;207(Suppl 2):S57-S62. doi:10.1093/infdis/jit111

10. Erickson DA, Mather G, Trager WF, Levy RH, Keirns JJ. Characterization of the in vitro biotransformation of the HIV-1 reverse transcriptase inhibitor nevirapine by human hepatic cytochromes P-450. Drug Metab Dispos. 1999;27(12):1488-1495.

11. Desta Z, Saussele T, Ward B, et al. Impact of CYP2B6 polymorphism on hepatic efavirenz metabolism in vitro. Pharmacogenomics. 2007;8 (6):547-558. doi:10.2217/14622416.8.6.547

12. Di Iulio J, Fayet A, Arab-Alameddine M, et al. In vivo analysis of efavirenz metabolism in individuals with impaired CYP2A6 function. Pharmacogenet Genomics. 2009;19(4):300-309. doi:10.1097/FPC.0b 013e328328d577

13. Alessandrini M, Pepper MS. Priority pharmacogenetics for the African continent: focus on CYP450. Pharmacogenomics. 2014;15 (3):385-400. doi:10.2217/pgs.13.252.

14. Russo G, Paganotti GM, Soeria-Atmadja S, et al. Pharmacogenetics of non-nucleoside reverse transcriptase inhibitors (NNRTIs) in resource-limited settings: influence on antiretroviral therapy response and concomitant anti-tubercular, antimalarial and contraceptive treatments. Infect Genet Evol. 2016;37:192-207. doi:10.1016/j. meegid.2015.11.014

15. Haas DW, Ribaudo HJ, Kim RB, et al. Pharmacogenetics of efavirenz and central nervous system side effects: an adult AIDS Clinical Trials Group study. AIDS. 2004;18(18):2391-2400. 
16. Ribaudo HJ, Haas DW, Tierney C, et al. Pharmacogenetics of plasma efavirenz exposure after treatment discontinuation: an adult AIDS Clinical Trials Group Study. Clin Infect Dis. 2006;42(3):401-407. doi: $10.1086 / 499364$

17. Mutwa PR, Fillekes Q, Malgaz M, et al. Mid-dosing interval efavirenz plasma concentrations in HIV-1-infected children in Rwanda: treatment efficacy, tolerability, adherence, and the influence of CYP2B6 polymorphisms. J Acquir Immune Defic Syndr. 2012;60 (4):400-404. doi:10.1097/QAI.0b013e3182569f57

18. Sarfo FS, Zhang Y, Egan D, et al. Pharmacogenetic associations with plasma efavirenz concentrations and clinical correlates in a retrospective cohort of Ghanaian HIV-infected patients. J Antimicrob Chemother. 2014;69(2):491-499. doi:10.1093/jac/ $\mathrm{dkt} 372$

19. Dickinson L, Chaponda M, Carr DF, et al. Population pharmacokinetic and pharmacogenetic analysis of nevirapine in hypersensitive and tolerant HIV-infected patients from Malawi. Antimicrob Agents Chemother. 2014;58(2):706-712. doi:10.1128/AAC.02069-13

20. Oluka MN, Okalebo FA, Guantai AN, McClelland RS, Graham SM. Cytochrome P450 2B6 genetic variants are associated with plasma nevirapine levels and clinical response in HIV-1 infected Kenyan women: a prospective cohort study. AIDS Res Ther. 2015;12:10. doi:10.1186/s12981-015-0052-0

21. Chen J, Sun J, Ma Q, et al. CYP2B6 polymorphism and nonnucleoside reverse transcriptase inhibitor plasma concentrations in Chinese HIV-infected patients. Ther Drug Monit. 2010;32(5):573-578. doi:10.1097/FTD.0b013e3181 ea953c

22. Lehr T, Yuan J, Hall D, et al. Integration of absorption, distribution, metabolism, and elimination genotyping data into a population pharmacokinetic analysis of nevirapine. Pharmacogenet Genomics. 2011;21(11):721-730. doi:10.1097/FPC.0b013e32834a522e

23. Zukunft J, Lang T, Richter T, et al. A natural CYP2B6 TATA box polymorphism $(-82 \mathrm{~T}->\mathrm{C})$ leading to enhanced transcription and relocation of the transcriptional start site. Mol Pharmacol. 2005;67 (5):1772-1782. doi:10.1124/mol.104.008086

24. Bumpus NN, Kent UM, Hollenberg PF. Metabolism of efavirenz and 8-hydroxyefavirenz by P450 2B6 leads to inactivation by two distinct mechanisms. J Pharmacol Exp Ther. 2006;318(1):345-351. doi:10. 1124/jpet.106.102525

25. Zanger UM, Klein K, Saussele T, Blievernicht J, Hofmann MH, Schwab M. Polymorphic CYP2B6: molecular mechanisms and emerging clinical significance. Pharmacogenomics. 2007;8(7):743-759. doi:10.2217/14622416.8.7.743

26. Wang J, Sönnerborg A, Rane A, et al. Identification of a novel specific CYP2B6 allele in Africans causing impaired metabolism of the HIV drug efavirenz. Pharmacogenet Genomics. 2006;16 (3):191-198. doi:10.1097/01.fpc.0000189797.03845.90

27. Bertrand J, Chou M, Richardson DM, et al. Multiple genetic variants predict steady-state nevirapine clearance in HIV-infected cambodians. Pharmacogenet Genomics. 2012;22(12):868-876. doi:10. 1097/FPC.0b013e32835a5af2

28. Desta Z, Gammal RS, Gong L, et al. Clinical Pharmacogenetics Implementation Consortium (CPIC) guideline for CYP2B6 and efavirenz-containing antiretroviral therapy. Clin Pharmacol Ther. 2019;106(4):726-733. doi:10.1002/cpt.147

29. Vujkovic M, Bellamy SL, Zuppa AF, et al. Brief report: CYP2B6 $516 \mathrm{G}>\mathrm{T}$ minor allele protective of late virologic failure in efavirenztreated HIV-infected patients in Botswana. J Acquir Immune Defic Syndr. 2017;75(4):488-491. doi:10.1097/QAI.0000000000001442

30. Gross R, Bellamy SL, Ratshaa B, et al. CYP2B6 genotypes and early efavirenz-based HIV treatment outcomes in Botswana. AIDS 2017;31(15):2107-2113. doi:10.1097/QAD.0000000000001593

31. Ribaudo HJ, Liu H, Schwab M, et al. Effect of CYP2B6, ABCB1, and CYP3A5 polymorphisms on efavirenz pharmacokinetics and treatment response: an AIDS Clinical Trials Group study. $J$ Infect Dis. 2010;202(5):717-722. doi:10.1086/655470
32. Frasco MA, Mack WJ, Van Den Berg D, et al. Underlying genetic structure impacts the association between CYP2B6 polymorphisms and response to efavirenz and nevirapine. AIDS. 2012;26 (16):2097-2106. doi:10.1097/QAD.0b013e3283593602

33. Haas DW, Smeaton LM, Shafer RW, et al. Pharmacogenetics of long-term responses to antiretroviral regimens containing efavirenz and/or nelfinavir: an Adult Aids Clinical Trials Group Study. J Infect Dis. 2005;192(11):1931-1942. doi:10.1086/497610

34. Haas DW, Severe P, Jean Juste MA, Pape JW, Fitzgerald DW. Functional CYP2B6 variants and virologic response to an efavirenz-containing regimen in Port-au-Prince, Haiti. J Antimicrob Chemother. 2014;69(8):2187-2190. doi:10.1093/jac/dku088

35. Lehmann DS, Ribaudo HJ, Daar ES, et al. Genome-wide association study of virologic response with efavirenz-containing or abacavir-containing regimens in AIDS clinical trials group protocols. Pharmacogenet Genomics. 2015;25(2):51-59. doi:10.10 97/FPC.0000000000000106

36. Rotger M, Colombo S, Furrer H, et al. Influence of CYP2B6 polymorphism on plasma and intracellular concentrations and toxicity of efavirenz and nevirapine in HIV-infected patients. Pharmacogenet Genomics. 2005;15(1):1-5. doi:10.1097/01213011-200501000-00001

37. Gounden V, van Niekerk C, Snyman T, George JA. Presence of the CYP2B6 516G $>$ T polymorphism, increased plasma efavirenz concentrations and early neuropsychiatric side effects in South African HIV-infected patients. AIDS Res Ther. 2010;7:32. doi:10.1186/17426405-7-32

38. Yuan J, Guo S, Hall D, et al. Toxicogenomics of nevirapine-associated cutaneous and hepatic adverse events among populations of African, Asian, and European descent. AIDS. 2011;25 (10):1271-1280. doi:10.1097/QAD.0b013e32834779df

39. Ciccacci C, Di Fusco D, Marazzi MC, et al. Association between CYP2B6 polymorphisms and nevirapine-induced SJS/TEN: a pharmacogenetics study. Eur J Clin Pharmacol. 2013;69(11): 1909-1916. doi:10.1007/s00228-013-1549-x

40. Mukonzo JK, Okwera A, Nakasujja N, et al. Influence of efavirenz pharmacokinetics and pharmacogenetics on neuropsychological disorders in Ugandan HIV-positive patients with or without tuberculosis: a prospective cohort study. BMC Infect Dis. 2013;13:261. doi:10.1186/1471-2334-13-261

41. Vujkovic M, Bellamy SL, Zuppa AF, et al. Polymorphisms in cytochrome $\mathrm{P} 450$ are associated with extensive efavirenz pharmacokinetics and CNS toxicities in an HIV cohort in Botswana. Pharmacogenomics J. 2018;18(5):678-688. doi:10.1038/s41397018-0028-2

42. Kwara A, Lartey M, Sagoe KW, Kenu E, Court MH. CYP2B6, CYP2A6 and UGT2B7 genetic polymorphisms are predictors of efavirenz mid-dose concentration in HIV-infected patients. AIDS. 2009;23(16):2101-2106. doi:10.1097/QAD.0b013e3283319908

43. Cummins NW, Neuhaus J, Chu H, et al. Investigation of efavirenz discontinuation in multi-ethnic populations of HIV-positive individuals by genetic analysis. EBioMedicine. 2015;2(7):706-712. doi:10.1016/j.ebiom.2015.05.012

44. Mukonzo JK, Owen JS, Ogwal-Okeng J, et al. Pharmacogeneticbased efavirenz dose modification: suggestions for an African population and the different CYP2B6 genotypes. PLoS One. 2014;9(1): e86919. doi:10.1371/journal.pone.0086919

45. Mhandire D, Lacerda M, Castel S, et al. Effects of CYP2B6 and CYP1A2 genetic variation on nevirapine plasma concentration and pharmacodynamics as measured by CD4 cell count in Zimbabwean HIV-infected patients. OMICS. 2015;19(9):553-562. doi:10.1089/ omi.2015.0104

46. Wester CW, Thomas AM, Bussmann H, et al. Non-nucleoside reverse transcriptase inhibitor outcomes among combination antiretroviral therapy-treated adults in Botswana. AIDS. 2010;24(Suppl 1 (Suppl 1)):S27-S36. doi:10.1097/01.aids.0000366080.91192.55 
47. Lavandera JV, Parera VE, Rossetti MV, Batlle AM, Buzaleh AM. Identification of CYP3A5 and CYP2B6 polymorphisms in porphyria cutanea tarda associated to human immunodeficiency virus. $J$ Clin Exp Dermatol Res. 2011;S2:006. doi:10.4172/2155-9554.S2-006.

48. Paganotti GM, Russo G, Sobze MS, et al. CYP2B6 poor metaboliser alleles involved in efavirenz and nevirapine metabolism: CYP2B $6 * 9$ and CYP2B6*18 distribution in HIV-exposed subjects from Dschang, Western Cameroon. Infect Genet Evol. 2015;35:122-126. doi:10.10 16/j.meegid.2015.08.003

49. Tawe L, Motshoge T, Ramatlho P, et al. Human cytochrome P450 2B6 genetic variability in Botswana: a case of haplotype diversity and convergent phenotypes. Sci Rep. 2018;8(1):4912. doi:10.1038/ s41598-018-23350-1

50. Kumondai M, Hosono H, Orikasa K, et al. Genetic polymorphisms of CYP2A6 in a case-control study on bladder cancer in Japanese smokers. Biol Pharm Bull. 2016;39(1):84-89. doi:10.1248/bpb.b1500604

51. Dodgen TM, Drögemöller BI, Wright GE, et al. Evaluation of predictive CYP2C19 genotyping assays relative to measured phenotype in a South African cohort. Pharmacogenomics. 2015;16(12):13 43-1354. doi:10.2217/pgs.15.80

52. Gaedigk A, Simon SD, Pearce RE, Bradford LD, Kennedy MJ, Leeder JS. The CYP2D6 activity score: translating genotype information into a qualitative measure of phenotype. Clin Pharmacol Ther. 2008;83(2):234-242. doi:10.1038/sj.clpt.6100406

53. Excoffier L, Lischer HE. Arlequin suite ver 3.5: a new series of programs to perform population genetics analyses under linux and windows. Mol Ecol Resour. 2010;10(3):564-567. doi:10.1111/j.17550998.2010.02847.x

54. Mackie N. Resistance to non-nucleoside reverse transcriptase inhibitors. In: Geretti AM, editor. Antiretroviral Resistance in Clinical Practice. London: Mediscript;2006:Chapter 2. Available from: https://www.ncbi. nlm.nih.gov/books/NBK2249/. Accessed October 1, 2020.

55. Paganotti GM, Gallo BC, Verra F, et al. Human genetic variation is associated with Plasmodium falciparum drug resistance. J Infect Dis. 2011;204(11):1772-1778. doi:10.1093/infdis/jir629

56. Cavaco I, Mårtensson A, Fröberg G, Msellem M, Björkman A, Gil JP. CYP2C8 status of patients with malaria influences selection of Plasmodium falciparum pfmdr1 alleles after amodiaquine-artesunate treatment. J Infect Dis. 2013;207(4):687-688. doi:10.1093/infdis/ jis736

57. Gross R, Aplenc R, Tenhave T, et al. Slow efavirenz metabolism genotype is common in Botswana. J Acquir Immune Defic Syndr. 2008;49(3):336-337. doi:10.1097/QAI.0b013e31817c1ed0

58. Arnaldo P, Thompson RE, Lopes MQ, Suffys PN, Santos AR. Frequencies of cytochrome P450 2B6 and 2C8 allelic variants in the Mozambican Population. Malays J Med Sci. 2013;20(4):13-23.

59. Cohen K, Grant A, Dandara C, et al. Effect of rifampicin-based antitubercular therapy and the cytochrome $\mathrm{P} 450$ 2B6 516G $>$ T polymorphism on efavirenz concentrations in adults in South Africa. Antivir Ther. 2009;14(5):687-695.

60. Swart M, Skelton M, Wonkam A, Kannemyer L, Chin'ombe N, Dandara C. CYP1A2, CYP2A6, CYP2B6, CYP3A4 and CYP3A5 polymorphisms in two bantu-speaking populations from cameroon and South Africa: implications of global pharmacogenetics. Curr Pharmacogenomics Person Med. 2012;10(1):43-53. doi:10.2174/ 1875692111201010043

61. Swart M, Skelton M, Ren Y, Smith P, Takuva S, Dandara C. High predictive value of CYP2B6 SNPs for steady-state plasma efavirenz levels in South African HIV/AIDS patients. Pharmacogenet Genomics. 2013;23(8):415-427. doi:10.1097/FPC.0b013e3283631 $76 \mathrm{f}$

62. Sinxadi PZ, Leger PD, McIlleron HM, et al. Pharmacogenetics of plasma efavirenz exposure in HIV-infected adults and children in South Africa. Br J Clin Pharmacol. 2015;80(1):146-156. doi:10.11 11/bcp. 12590
63. Röhrich CR, Drögemöller BI, Ikediobi O, et al. CYP2B6*6 and CYP2B6*18 predict long-term efavirenz exposure measured in hair samples in HIV-positive South African women. AIDS Res Hum Retroviruses. 2016;32(6):529-538. doi:10.1089/AID.2015.0048

64. Reay R, Dandara C, Viljoen M, Rheeders M. CYP2B6 haplotype predicts efavirenz plasma concentration in Black South African HIV-1-infected children: a Longitudinal Pediatric Pharmacogenomic Study. OMICS. 2017;21(8):465-473. doi:10.1089/omi.2017.0078

65. Jamshidi Y, Moreton M, McKeown DA, et al. Tribal ethnicity and CYP2B6 genetics in Ugandan and Zimbabwean populations in the UK: implications for efavirenz dosing in HIV infection. $J$ Antimicrob Chemother. 2010;65(12):2614-2619. doi:10.1093/jac/dkq369

66. Nyakutira C, Röshammar D, Chigutsa E, et al. High prevalence of the CYP2B6 516G->T(*6) variant and effect on the population pharmacokinetics of efavirenz in HIV/ AIDS outpatients in Zimbabwe. Eur $J$ Clin Pharmacol. 2008;64(4):357-365. doi:10.1007/s00228-0070412-3

67. Maimbo M, Kiyotani K, Mushiroda T, Masimirembwa C, Nakamura Y. CYP2B6 genotype is a strong predictor of systemic exposure to efavirenz in HIV-infected Zimbabweans. Eur J Clin Pharmacol. 2012;68(3):267-271. doi:10.1007/s00228-011-1118-0

68. Dhoro M, Zvada S, Ngara B, et al. CYP2B6*6, CYP2B6*18, body weight and sex are predictors of efavirenz pharmacokinetics and treatment response: population pharmacokinetic modeling in an HIV/ AIDS and TB cohort in Zimbabwe. BMC Pharmacol Toxicol. 2015;16(4). doi:10.1186/s40360-015-0004-2

69. Nguefeu Nkenfou C, Atogho Tiedeu B, Nguefeu Nkenfou C, et al. Adverse drug reactions associated with CYP 2B6 polymorphisms in HIV/ AIDS-treated patients in Yaoundé, Cameroon. Appl Clin Genet. 2019;12:261-268. doi:10.2147/TACG.S226318

70. Klein K, Lang T, Saussele T, et al. Genetic variability of CYP2B6 in populations of African and Asian origin: allele frequencies, novel functional variants, and possible implications for anti-HIV therapy with efavirenz. Pharmacogenet Genomics. 2005;15(12):861-873. doi:10.1097/01213011-200512000-00004

71. Mehlotra RK, Ziats MN, Bockarie MJ, Zimmerman PA. Prevalence of CYP2B6 alleles in malaria-endemic populations of West Africa and Papua New Guinea. Eur J Clin Pharmacol. 2006;62(4):267-275. doi:10.1007/s00228-005-0092-9

72. Mehlotra RK, Bockarie MJ, Zimmerman PA. CYP2B6 983T $>$ C polymorphism is prevalent in West Africa but absent in Papua New Guinea: implications for HIV/AIDS treatment. Br J Clin Pharmacol. 2007;64 (3):391-395. doi:10.1111/j.1365-2125.2007.02884.x

73. Kwara A, Lartey M, Sagoe KW, Rzek NL, Court MH. CYP2B6 $($ c.516G $->\mathrm{T})$ and CYP2A6 (*9B and/or *17) polymorphisms are independent predictors of efavirenz plasma concentrations in HIVinfected patients. Br J Clin Pharmacol. 2009;67(4):427-436. doi:10.1111/j.1365-2125.2009.03368.x

74. Sa'ad Toyin A, Julius Olugbenga S, Rahman Ayodele B, Olusola Joseph O, Taibat Moji BO. Allele and genotype frequencies of cytochrome P450 2B6 516G $>$ T single nucleotide polymorphism in HIVnegative and HIV-infected adult Nigerian Populations. Am J Ther. 2016;23(6):e1715-e1719. doi:10.1097/MJT.0000000000000340

75. Olagunju A, Schipani A, Bolaji O, Khoo S, Owen A. Evaluation of universal versus genotype-guided efavirenz dose reduction in pregnant women using population pharmacokinetic modelling. J Antimicrob Chemother. 2018;73(1):165-172. doi:10.1093/jac/dkx334

76. Peko SM, Gueye NSG, Vouvoungui C, et al. Cytochrome P450 CYP2B6*6 distribution among CONGOLESE individuals with HIV, tuberculosis and malaria infection. Int J Infect Dis. 2019;82:111-116. doi:10.1016/j.ijid.2019.02.025

77. Calcagno A, D'Avolio A, Simiele M, et al. Influence of CYP2B6 and ABCB1 SNPs on nevirapine plasma concentrations in burundese HIV-positive patients using dried sample spot devices. Br J Clin Pharmacol. 2012;74(1):134-140. doi:10.1111/j.1365-2125.2012.041 63. $\mathrm{x}$ 
78. Habtewold A, Amogne W, Makonnen E, et al. Long-term effect of efavirenz autoinduction on plasma/peripheral blood mononuclear cell drug exposure and CD4 count is influenced by UGT2B7 and CYP2B6 genotypes among HIV patients. J Antimicrob Chemother. 2011;66(10):2350-2361. doi:10.1093/jac/dkr304

79. Yimer G, Amogne W, Habtewold A, et al. High plasma efavirenz level and CYP2B6*6 are associated with efavirenz-based HAARTinduced liver injury in the treatment of naïve HIV patients from Ethiopia: a prospective cohort study. Pharmacogenomics J. 2012;12 (6):499-506. doi:10.1038/tpj.2011.34

80. Ngaimisi E, Habtewold A, Minzi O, et al. Importance of ethnicity, CYP2B6 and ABCB1 genotype for efavirenz pharmacokinetics and treatment outcomes: a parallel-group prospective cohort study in two sub-Saharan Africa populations. PLoS One. 2013;8(7):e67946. doi:10.1371/journal.pone.0067946

81. Habtewold A, Aklillu E, Makonnen E, et al. Population pharmacokinetic model linking plasma and peripheral blood mononuclear cell concentrations of efavirenz and its metabolite, 8-hydroxy-efavirenz, in HIV patients. Antimicrob Agents Chemother. 2017;61(8):e00207e00217. doi:10.1128/AAC.00207-17

82. Radloff R, Gras A, Zanger UM, et al. Novel CYP2B6 enzyme variants in a Rwandese population: functional characterization and assessment of in silico prediction tools. Hum Mutat. 2013;34 (5):725-734. doi:10.1002/humu.22295

83. Bienvenu E, Swart M, Dandara C, Ashton M. The role of genetic polymorphisms in cytochrome $\mathrm{P} 450$ and effects of tuberculosis co-treatment on the predictive value of CYP2B6 SNPs and on efavirenz plasma levels in adult HIV patients. Antiviral Res. 2014; 102:44-53. doi:10.1016/j.antiviral.2013.11.011

84. Marwa KJ, Schmidt T, Sjögren M, Minzi OM, Kamugisha E, Swedberg G. Cytochrome P450 single nucleotide polymorphisms in an indigenous Tanzanian population: a concern about the metabolism of artemisinin-based combinations. Malar J. 2014;13:420. doi:10. $1186 / 1475-2875-13-420$
85. Maganda BA, Minzi OM, Ngaimisi E, Kamuhabwa AA, Aklillu E. CYP2B6*6 genotype and high efavirenz plasma concentration but not nevirapine are associated with low lumefantrine plasma exposure and poor treatment response in HIV-malaria-coinfected patients. Pharmacogenomics J. 2016;16(1):88-95. doi:10.1038/ tpj.2015.37

86. Mutagonda RF, Kamuhabwa AAR, Minzi OMS, et al. Effect of pharmacogenetics on plasma lumefantrine pharmacokinetics and malaria treatment outcome in pregnant women. Malar J. 2017;16 (1):267. doi:10.1186/s12936-017-1914-9

87. Kitabi EN, Minzi OMS, Mugusi S, et al. Long-term efavirenz pharmacokinetics is comparable between Tanzanian HIV and HIV/tuberculosis patients with the same CYP2B6*6 genotype. Sci Rep. 2018;8 (1):16316. doi:10.1038/s41598-018-34674-3

88. Penzak SR, Kabuye G, Mugyenyi P, et al. Cytochrome P450 2B6 (CYP2B6) G516T influences nevirapine plasma concentrations in HIV-infected patients in Uganda. HIV Med. 2007;8(2):86-91. doi:10.1111/j.1468-1293.2007.00432.x

89. Mukonzo JK, Röshammar D, Waako P, et al. A novel polymorphism in ABCB1 gene, CYP2B6*6 and sex predict single-dose efavirenz population pharmacokinetics in Ugandans. $\mathrm{Br} J$ Clin Pharmacol. 2009;68(5):690-699. doi:10.1111/j.1365-2125.2009.03516.x

90. von Braun A, Castelnuovo B, Ledergerber B, et al. High efavirenz serum concentrations in TB/HIV-coinfected Ugandan adults with a CYP2B6 516 TT genotype on anti-TB treatment. J Antimicrob Chemother. 2019;74(1):135-138. doi:10.1093/jac/dky379

91. Neary M, Chappell CA, Scarsi KK, et al. Effect of patient genetics on etonogestrel pharmacokinetics when combined with efavirenz or nevirapine ART. J Antimicrob Chemother. 2019;74(10):3003-3010. doi:10.1093/jac/dkz298
Pharmacogenomics and Personalized Medicine

\section{Publish your work in this journal}

Pharmacogenomics and Personalized Medicine is an international, peer-reviewed, open access journal characterizing the influence of genotype on pharmacology leading to the development of personalized treatment programs and individualized drug selection for improved safety, efficacy and sustainability. This journal is indexed on the American Chemical Society's Chemical Abstracts Service (CAS). The manuscript management system is completely online and includes a very quick and fair peer-review system, which is all easy to use. Visit http://www.dovepress.com/testimonials.php to read real quotes from published authors. 